\title{
Cyclostationary Modelling of Reciprocating Compressors and Application to Valve Fault Detection
}

\author{
R. Zouari \\ Institut de Recherche en Informatique et Systèmes Alèatoires, \\ Campus universitaire de Beaulieu, F-35042 Rennes cedex, France
}

\author{
J. Antoni, J. L. Ille and M. Sidahmed
}

Universitè de Technologie de Compiègne, France

\author{
M. Willaert \\ Burton Corblin, Nogent-sur-Oise, France
}

\section{Watremetz \\ Centre Technique des Industries Mècaniques, Senlis, France}

(Received 3 June 2006; accepted 19 June 2007)

\begin{abstract}
This paper presents a methodology for identifying events and valve faults in reciprocating compressors based on cyclostationary modelling of their vibration. The discussion in this paper first concerns the resampling of the time signals and then their decomposition into periodic and random parts. Secondly, the second-order cyclostationarity of the random part is exploited to construct an angular frequency map of the vibration energy by means of the Wigner-Ville spectrum based on cyclic averaging. From this energy representation, a correlation procedure between sensors is followed in order to identify the angular and spectral bands where coherent events occur in the compressor cycle. Additionally, it allows the extraction of pertinent and simple indicators for detecting fault signatures in valves; these can be used in condition monitoring.
\end{abstract}

${ }^{\dagger}$ Member of the International Institute of Acoustics and Vibration (IIAV)

\section{INTRODUCTION}

The use of signal processing tools in the condition monitoring of machines is a topic of growing potential and interest in industry. In fact with economical and nonintrusive instrumentation, signal processing can provide useful information about the identification of the physical phenomena (observability), and the detection, and the isolation of faults (diagnostics). For rotating and reciprocating machines, classical maintenance techniques are commonly based on oil, gas and temperature analyses (diesel engines), pressure (pumps) or electrical measurements (asynchronous machines); more recently, acoustic emission has shown an interesting potential. ${ }^{1}$ But no doubt, the most used technique is still vibration analysis which provides the best sensitivity to the dynamics and kinematics of such machines. The choice of a vibrationmonitoring analysis procedure should be made in accordance with the operational characteristic of the machine. Conventional approaches, such as Fourier or ARMA analyses, are based on the assumptions that vibration signals are stationary (having time invariant statistics) and ergodic (statistical indicators can be computed from time averages); this is usually a legitimate assumption for rotating machines. However, when coming to reciprocating machines such as IC engines and compressors, the dissipation of energy is strongly unsteady due to shocks and abrupt pressure changes, thus resulting in vibration signals that are transient and nonstationary (having time variant statistics). Even though no theory can universally model all nonstationary processes, some dedicated tools such as envelope analysis and time-frequency representation are efficient enough to follow energy changes and to detect intermittent faults. When applied to reciprocating machines, these representations show that the energy changes are periodic, in synchronisation with the machine operating cycle, so that the process can be considered as cyclostationary.

Even though cyclostationarity is a concept that has appeared in wireless communication engineering, ${ }^{2}$ its application to machine condition monitoring has only recently witnessed fruitful successes. ${ }^{3-5}$ However, taking advantage of the cyclostationarity of a machine requires the introduction of specific signal preprocessing algorithms, especially to cancel out any rotational velocity fluctuations. In fact, to maintain the same number of samples for all recorded cycles of a machine, time signals are usually resampled according to an angular reference locked to the machine kinematics; therefore, all statistical averages necessary to compute monitoring indicators can be performed in a cyclic manner (having synchronous statistics). ${ }^{6,7}$ Many condition monitoring applications have shown that, compared to conventional approaches, cyclostationary modelling and cyclic signal processing yield extra information that greatly helps to better characterise physi- 